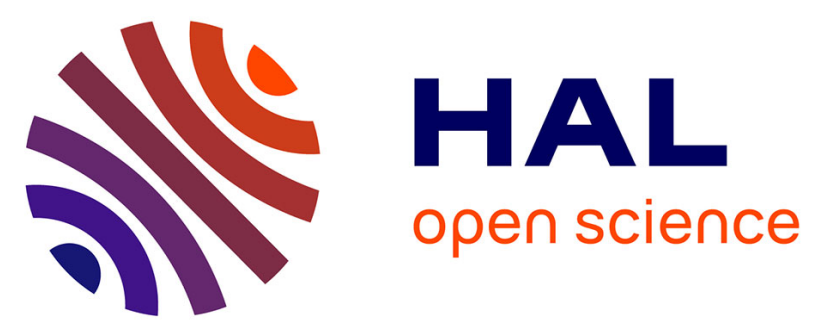

\title{
Etude de deux nouvelles sources de résistance à Phytophthora capsici Leon. chez le piment : confirmation de l'existence de trois composantes distinctes dans la résistance
}

\author{
E. Pochard, Paul-Michel Molot, Gabriel Dominguez
}

\section{To cite this version:}

E. Pochard, Paul-Michel Molot, Gabriel Dominguez. Etude de deux nouvelles sources de résistance à Phytophthora capsici Leon. chez le piment : confirmation de l'existence de trois composantes distinctes dans la résistance. Agronomie, 1983, 3 (4), pp.333-342. 10.1051/agro:19830406 . hal-02728349

\section{HAL Id: hal-02728349 \\ https://hal.inrae.fr/hal-02728349}

Submitted on 2 Jun 2020

HAL is a multi-disciplinary open access archive for the deposit and dissemination of scientific research documents, whether they are published or not. The documents may come from teaching and research institutions in France or abroad, or from public or private research centers.
L'archive ouverte pluridisciplinaire HAL, est destinée au dépôt et à la diffusion de documents scientifiques de niveau recherche, publiés ou non, émanant des établissements d'enseignement et de recherche français ou étrangers, des laboratoires publics ou privés. 


\title{
Etude de deux nouvelles sources de résistance à Phytophthora capsici Leon. chez le piment: confirmation de l'existence de trois composantes distinctes dans la résistance.
}

\author{
Edmond POCHARD, Paul-Michel MOLOT $\left({ }^{*}\right)$ \& Gabriel DOMINGUEZ (**) \\ I.N.R.A., Station d'Amélioration des Plantes maraîchères, \\ (*) Station de Pathologie végétale, Centre de Recherches d'Avignon, F 84140 Montfavet. \\ (**) Facultad de Ciencias Agronomicas y Veterinarias, Estación Experimental Engombe, Santo Domingo, \\ République Dominicaine.
}

L'étude de l'évolution de la vitesse de nécrose des tiges chez 4 génotypes de piment, dont 2 lignées reçues du Mexique, permet de confirmer l'existence d'au moins 3 composantes distinctes dans la résistance du Capsicum à Phytophthora capsici. Ces composantes ont été précédemment désignées par les termes : " réceptivité ", « inductibilité » et « stabilité " (PoCHARD \& DAuBEze, 1980). Par ailleurs, l'effet de hautes températures continues $\left(32^{\circ} \mathrm{C}\right)$ sur chacune de ces composantes est précisé.

La lignée «Criollo de Morelos 334 » est remarquable par sa très faible réceptivité vis-à-vis des 4 souches utilisées, à $22^{\circ} \mathrm{C}$ comme à $32^{\circ} \mathrm{C}:$ la vitesse initiale de nécrose de la tige est réduite en moyenne de $30 \mathrm{p} .100$ $\left(22^{\circ} \mathrm{C}\right)$ et 50 p. $100\left(32^{\circ} \mathrm{C}\right)$ par rapport à celle des autres variétés.

La lignée « L 29 » montre une très forte aptitude à l'induction : la décélération de la vitesse de nécrose est plus forte que celle des autres lignées quelle que soit la souche ou la température étudiée, entre le $3^{e}$ et le $5^{\mathrm{e}}$ ou le $7^{\mathrm{e}}$ jour après l'infection.

L'un des éléments de la $3^{\mathrm{e}}$ composante définie antérieurement comme la « stabilité de la résistance 》 apparaît sous la forme d'un « potentiel de résistance » caractéristique du génotype considéré, peu ou pas modifié par la température. La lignée « PM 217 » montre un potentiel de résistance particulièrement élevé : elle est capable, malgré sa faible inductibilité, de ramener la vitesse de pénétration du champignon dans la tige à des valeurs très faibles, même si la vitesse initiale est très forte sous l'influence d'une souche très agressive et/ou d'une température très élevée. Au contraire «Phyo 636 " a un potentiel de résistance peu élevé, évalué à moins de la moitié de celui de son parent d'origine «PM 217 ».

Mots-clés additionnels : Résistance polygénique, Composantes de la résistance, Température, Souches, Capsicum annuum. ther evidence for the existence of three resistance components.

Two Capsicum annuum lines of Mexican origin were studied at two temperatures $\left(22^{\circ}\right.$ and $\left.32^{\circ} \mathrm{C}\right)$ and compared with "PM 217 » and « Phyo 636 », already described, for their reaction to Phytophthora capsici strains. Rate of stem necrosis was recorded twice a week for 3 weeks (Pochard \& DAUBEZE, 1980).

The 3 components (or dimensions) of the resistance described in a previous publication and termed : « receptivity ", " inducibility » and « stability » were easily identified, each variety being remarkable for a particular point. Line « Criollo de Morelos 334 " showed very low receptivity (low initial rate of stem necrosis) whatever the strain or temperature used : $30 \%\left(22^{\circ} \mathrm{C}\right)$ and $50 \%\left(32^{\circ} \mathrm{C}\right)$ reduction compared with the other varieties. Line « L 29 " showed a better inducibility than the other genotypes (lower rate of necrosis between the 3rd and 5 th or 7 th day) for all the temperature and strain combinations.

One of the constitutive elements of the third component of resistance previously termed « stability » appeared as a « resistance potential » characteristic of a given genotype. In spite of its low inducibility, line « PM 217 " showed the highest « resistance potential », i.e. the best ability to decrease the rate of stem necrosis to low levels even if the initial rate was very high (effect of a very aggressive strain and/or high temperature). In contrast, « Phyo 636 » had a low resistance potential, less than half that of its original parent « PM 217 ».

Additional key-words : Polygenic resistance, Components of resistance, Temperature, Strains, Capsicum annuum. 


\section{INTRODUCTION}

Les travaux conduits en France sur le couple piment Phytophthora capsici ont porté essentiellement sur les descendants du matériel original «PI 201234 » découvert il y a plus de 20 ans par KIMBLE \& GROGAN (1960). D'après les travaux de SMITH et al. (1967), la résistance serait contrôlée par 1 ou 2 gènes partiellement dominants suivant les lignées utilisées. Cette hypothèse est basée sur le dénombrement des plantes survivantes dans les générations de ségrégation lorsque les témoins sensibles sont entièrement détruits. Cependant, la mortalité se poursuit après cette date. De plus, les tests sont peu reproductibles. La lignée " PM 217 », de la collection de l'I.N.R.A. à Montfavet, est issue de ce même matériel mais les études quantitatives à l'échelle de la plante font apparaître un contrôle polygénique de la résistance. Celle-ci résulterait de la superposition d'au moins 3 mécanismes distincts (POCHARD \& DAUBEZE, 1980). De même, la variation du pouvoir pathogène des souches de champignon apparaît continue (CLERJEAU et al., 1976). Il est donc vain de vouloir définir des pathotypes puisqu'il n'existe pas de discontinuités objectives. C'est seulement sur des bases quantitatives que l'on peut espérer décrire les éléments de la relation complexe qui s'établit entre la plante et le Phytophthora.

Nous savons que l'essentiel de la résistance portée par « PM 217 » résulte d'une induction et n'est pas préexistant à l'infection. Nous avons appelé « inductibilité » l'aptitude à réduire la vitesse de pénétration du champignon dans la tige. Pratiquement, c'est entre le $3^{\mathrm{e}}$ et le $7^{\mathrm{e}}$ jour après l'inoculation que cette inductibilité apparaît le plus nettement dans nos conditions de test (PoCHARD et al., 1976; POCHARD \& DAUBEZE, 1980). D'après les travaux de Molot et al. (1981), c'est au $4^{\mathrm{e}}$ jour que la quantité de capsidiol est la plus importante dans les tiges à la température de $22^{\circ} \mathrm{C}$. Cependant, des essais de réinoculation montrent que d'autres phénomènes n'impliquant pas cette phytoalexine jouent un rôle important dans les phases plus tardives que la résistance (MOLOT et al., 1980). Pour un ensemble de souches, les valeurs d'inductibilité varient chez les diverses lignées tirées du géniteur «PM 217 » par recroisements successifs à des variétés sensibles (PocHARD \& Daubeze, 1980).

Cette relation centrale est flanquée de 2 autres modes de relation qui sont passés tout d'abord inaperçus mais qui jouent un grand rôle dans l'efficacité finale de la résistance. Pour une même masse d'inoculum et pour une souche donnée, la vitesse initiale de progression du champignon dans la tige, avant que le processus d'induction ne soit complètement fonctionnel, varie suivant les génotypes. Nous avons appelé « réceptivité » l'aptitude des tissus de la tige à offrir un substrat favorable à la croissance et/ou à la diffusion du champignon. Elle est préexistante à l'infection et varie en fonction de l'âge et du conditionnement préalable sous l'influence de facteurs du milieu. On cherche à la rendre la plus faible possible tout au long de la vie de la plante par des moyens génétiques ou culturaux.

La phase de ralentissement linéaire caractéristique des piments résistants peut se poursuivre plus ou moins longtemps. Lorsque la vitesse de progression du champignon descend en-dessous de $0,5 \mathrm{~mm}$ par jour, il s'établit un équilibre qui peut être durable entre la plante et l'agent pathogène, la vitesse d'invasion des tissus tendant lentement vers 0 , ou, au contraire, s'avérer fugace et laisser place à une nouvelle croissance rapide du champignon. Le comportement varie suivant les génotypes. Cette propriété a été dési- gnée par le terme de "stabilité » et fait référence à l'efficacité de la protection induite dans les tissus non encore envahis par le Phytophthora (effet intense ou non, durable ou non).

L'objet du présent travail est de caractériser, suivant les mêmes critères, 2 lignées d'origine mexicaine, données pour posséder une résistance plus efficace que les variétés issues du matériel de SMITH et al., vis-à-vis d'un grand nombre de souches (GUERRERO-MORENO \& LABORDE, 1980). De précédentes études ayant montré que la résistance de la lignée « Phyo 636 », issue de « PM 217 », était inefficace à une température continue supérieure à $28^{\circ} \mathrm{C}$ (POCHARD et al., 1976), nous avons répété les tests à 2 températures très différentes afin de déceler une éventuelle aptitude de ces lignées à supporter des températures supra-normales sans que la résistance soit trop affectée.

\section{MATÉRIEL \& MÉTHODES}

\section{A. Matériel végétal}

Le matériel végétal est constitué par 4 lignées, d'une part «PM 217 » et « Phyo 636 », servant de témoins, d'autre part « L 29 » et «CM 334 », échantillons fournis par A. Guerrero-Moreno, sélectionnés pour leur résistance à un grand nombre de souches mexicaines. " Phyo 636 » est tirée de «PM 217 » par recroisements successifs à la variété sensible «Yolo Wonder 》 puis autofécondations répétées, mais ne possède pas tous les éléments de résistance contenus dans le géniteur d'origine, surtout en ce qui concerne la stabilité de la résistance (PoCHARD \& DAUBEZE, 1980). « L 29 » est une lignée très précoce à fruit blancivoire ; «CM 334 » provient de « Criollo de Morelos ». Elle est de type " Serrano », très tardive, à tiges très velues et à fruits striés de violet (GUERRERO \& LABORDE, 1980.

\section{B. Matériel cryptogamique}

Quatres souches sont mises en comparaison. La souche 101 est la plus couramment utilisée dans les programmes de sélection. D'une agressivité moyenne, elle permet une très bonne discrimination entre «Phyo 636 » et les variétés sensibles à Phytophthora grâce à ses fortes capacités inductrices. La souche 107 est la plus agressive actuellement connue parmi celles qui ont été isolées en France : c'est avec elle que l'on observe les plus grandes longueurs de nécrose, dès le $3^{\mathrm{e}}$ jour après l'inoculation, chez les variétés sensibles (CleRJEAU et al., 1976). La souche 197 est considérée comme la plus dangereuse. Isolée sur «Phyo 636 » dans une serre où cette variété servait de porte-greffe, elle est à la fois agressive et peu inductrice de résistance. La souche 73 , isolée sur aubergine, est également très agressive sur piment ; elle semble différer de la souche 197 par de nombreuses propriétés in vivo et in vitro. La souche monozoospore 15-12 A est utilisée dans l'un des essais.

\section{Conditions de température et d'éclairement}

Deux températures ont été utilisées, maintenues constantes tout au long de l'expérience. L'une des chambres de culture était réglée à $22^{\circ} \mathrm{C}$ : c'est la température la plus couramment utilisée dans les tests de routine ; elle peut varier de $1^{\circ} \mathrm{C}$ autour de la valeur affichée. L'autre était réglée à $32^{\circ} \mathrm{C}$ (la température oscillait entre $30^{\circ}$ et $33^{\circ} \mathrm{C}$ ). L'éclai- 
rement était donné par des tubes luminescents et des lampes à filament donnant 50 à $60 \mathrm{~W} \cdot \mathrm{m}^{-2}$ au niveau des plantes pendant $12 \mathrm{~h}$.

\section{Dispositif expérimental}

Les plantes sont élevées en serre jusqu'à l'approche de la floraison des lots les plus précoces (« L 29 » et «PM 217 »). Les tiges sont décapitées sous la dernière feuille complètement étalée. L'inoculum est apporté sous forme de disque de culture pure recouvrant toute la section. Une feuille d'aluminium permet de maintenir une forte humidité à l'extrémité de la tige en formant une chambre humide.

La variable étudiée est la longueur de la nécrose externe induite par le champignon au fur et à mesure de sa progression dans la tige. On sait que cette progression s'effectue à une vitesse pratiquement constante chez les variétés sensibles, alors que chez les résistantes, après un départ plus ou moins rapide, on note un ralentissement progressif. Les mesures sont effectuées 2 fois par semaine pendant 3 semaines, à des intervalles de 3 et 4 jours, plus rapprochés à $32^{\circ} \mathrm{C}$. La vitesse moyenne d'invasion de la tige est calculée sur ces mêmes intervalles et rapportée au dernier jour de la période considérée.

Le dispositif choisi est de type split-plot, à 12 répétitions d'une plante par parcelle élémentaire à $22^{\circ} \mathrm{C}$ et 6 répétitions à $32^{\circ} \mathrm{C}$. L'effet principal est lié aux variétés, l'effet des différentes souches étant classé en $2^{\mathrm{e}}$ ordre.

\section{E. Mesure de la quantité de capsidiol produite dans la tige}

Parallèlement, un essai a été conduit pour estimer, par un test biologique, la quantité de capsidiol présente 4 jours après l'infection par la souche 15-12A dans le secteur de la tige situé juste en avant du front mycélien. Ce secteur est déterminé par des isolements mycologiques. L'extrait de tige est chromatographié en couches minces. La révélation biologique de la zone d'inhibition correspondant à la présence du capsidiol est effectuée par une pulvérisation de spores de Cladosporium herbarum; le diamètre de cette zone est proportionnel à la quantité de capsidiol (MOLOT et al., 1976 ; MOLOT et al., 1981).

\section{RÉSULTATS}

\section{A. Différences variétales de réceptivité et effet des souches}

La vitesse moyenne de nécrose de la tige pendant les 3 premiers jours, critère retenu pour estimer la réceptivité, est reportée dans le tableau 1. Il apparaît clairement que « C.M. 334 » a une réceptivité très inférieure à celle des 3 autres variétés, quelle que soit la souche ou la température. On remarque que la vitesse de progression du champignon n'est pas modifiée par une augmentation de la température de $10^{\circ} \mathrm{C}$, alors que, chez les autres variétés, elle est augmentée de 27 à 46 p. 100, «Phyo 636 » étant la plus affectée par les hautes températures.

L'effet de la température sur la vitesse de nécrose des tiges a été étudié précédemment chez la variété sensible «Yolo Wonder " pour les intervalles $16^{\circ}-22^{\circ} \mathrm{C}$ et $22^{\circ}-28^{\circ} \mathrm{C}$. $\mathrm{L}^{\prime}$ augmentation de la vitesse, ramenée à un intervalle théorique de $10^{\circ} \mathrm{C}$, était de 42 p. 100 et 87 p. 100 respectivement (PoCHARD et al., 1976).

En ce qui concerne les souches, on constate que la souche 101 est nettement moins agressive que les 3 autres aux 2 températures. On remarque que les effets additifs variétés et souches sont beaucoup plus importants que les effets d'interaction variété $x$ souche. A $22^{\circ} \mathrm{C}$, la variance de l'interaction est 16 et 32 fois plus faible que les variances liées aux variétés et aux souches. A $32^{\circ} \mathrm{C}$, ces coefficients sont respectivement 32 et 29.

\section{B. Différences variétales d'inductibilité et effet des souches}

L’inductibilité est estimée par la décélération de la vitesse de nécrose entre les $3^{\mathrm{e}}$ et $7^{\mathrm{e}}$ jours suivant l'inoculation à $22^{\circ} \mathrm{C}$, les $3^{\mathrm{e}}$ et $5^{\mathrm{e}}$ jours à $32^{\circ} \mathrm{C}$. Les résultats observés figurent au tableau 2.

Il apparaît que « L 29 » est beaucoup plus inductible que les autres variétés pour toutes les combinaisons de souches et de températures. Viennent ensuite « CM 334 » et « Phyo 636 ». " PM 217 » est classé dernier aux 2 températures.

La souche 101 n'est pas comparable aux autres car elle

TABLEAU 1

Evaluation de la réceptivité des variétés en présence de 4 souches, à 2 températures : propriétés exceptionnelles de «CM 334 》 comparée aux autres variétés (R).

Vitesse moyenne de nécrose de l'extrémité de la tige en $\mathrm{mm} \cdot \mathrm{j}^{-1}$ pendant les 3 premiers jours.

Test $F$ de Snedecor pour chaque combinaison de souches et de température : la probabilité des écarts observés entre les variétés à l'essai partout inférieure à $1 \mathrm{p} .1000$.

Evaluation of the varietal receptivity to 4 different strains at 2 temperatures : very low receptivity of "CM 334 " compared with the other resistant varieties (R). Mean speed of stem necrosis in mm.day ${ }^{-1}$ during the first 3 days.

$F$ test : probability of varietal differences less than 0.001 for all strains and temperatures.

\begin{tabular}{|c|c|c|c|c|c|c|c|c|c|c|c|}
\hline \multirow[b]{2}{*}{ Variétés } & \multicolumn{5}{|c|}{$22^{\circ} \mathrm{C}$} & \multicolumn{5}{|c|}{$32^{\circ} \mathrm{C}$} & \multirow{2}{*}{$\begin{array}{c}\text { Rapport } \\
\text { H/N }\end{array}$} \\
\hline & S 101 & S 73 & S 107 & S 197 & $\begin{array}{l}\text { Moyenne } \\
\text { (N) }\end{array}$ & S 101 & S 73 & S 107 & S 197 & $\begin{array}{l}\text { Moyenne } \\
(\mathrm{H})\end{array}$ & \\
\hline Phyo 636 & 5,64 & 9,50 & 9,08 & 8,94 & 8,29 & 7,78 & 13,67 & 13,56 & 13,50 & 12,13 & 1,46 \\
\hline PM 217 & 5,69 & 10,53 & 9,72 & 8,06 & 8,50 & 6,56 & 13,72 & 14,00 & 11,33 & 11,38 & 1,34 \\
\hline L 29 & 4,81 & 10,39 & 9,53 & 8,66 & 8,40 & 5,44 & 12,44 & 12,22 & 12,67 & 10,66 & 1,27 \\
\hline CM 334 & 4,08 & 7,03 & 5,89 & 5,86 & 5,72 & 3,28 & 7,28 & 6,33 & 5,67 & 5,64 & 0,99 \\
\hline $\begin{array}{l}\text { CM 334/R } \\
\text { Test F }\end{array}$ & $\begin{array}{l}0,76 \\
12.6\end{array}$ & $\begin{array}{c}0,72 \\
36.5\end{array}$ & $\begin{array}{c}0,62 \\
35.7\end{array}$ & $\begin{array}{c}0,68 \\
33.6\end{array}$ & 0,70 & $\begin{array}{l}0,50 \\
25,3\end{array}$ & $\begin{array}{c}0,55 \\
25.8\end{array}$ & $\begin{array}{c}0,48 \\
27.7\end{array}$ & $\begin{array}{c}0,45 \\
49.7\end{array}$ & 0,50 & \\
\hline
\end{tabular}




\section{TABLEAU 2}

Evaluation de l'inductibilité des variétés en présence des 3 souches les plus agressives. Décélération de la vitesse de nécrose de la tige entre le $3^{\mathrm{e}}$ et le $7^{\mathrm{e}}$ jour $\left(22^{\circ} \mathrm{C}\right)$, le $3^{\mathrm{e}}$ et le $5^{e}$ jour $\left(32^{\circ} \mathrm{C}\right)$.

Test de Duncan pour les comparaisons entre variétés et entre souches à chaque température, au seuil de probabilité 0,01 .

La lignée " $L 29$ » est plus inductible que les autres variétés; la souche 197 a de moins bonnes capacités d'induction.

Evaluation of the varietal inductibility in the presence of the 3 most aggressive strains.

Deceleration of the speed of stem necrosis between the $3 \mathrm{rd}$ and the 7 th day $\left(22^{\circ} \mathrm{C}\right), 3 \mathrm{rd}$ and 5 th day $\left(32^{\circ} \mathrm{C}\right)$; Duncan test for the comparison of varieties and strains, at the level 0.01 ; line * L 29 " appears more inductible than the other resistant varieties; strain 197 has a weaker induction capacity.

\begin{tabular}{|c|c|c|c|c|c|c|c|c|c|}
\hline \multirow[b]{2}{*}{ Variétés } & \multicolumn{4}{|c|}{$22^{\circ} \mathrm{C}$} & \multicolumn{4}{|c|}{$32^{\circ} \mathrm{C}$} & \multirow{2}{*}{$\begin{array}{c}\text { Rapport } \\
\text { H/N }\end{array}$} \\
\hline & S 73 & S 107 & S 197 & $\begin{array}{l}\text { Moyenne } \\
\text { (N) }\end{array}$ & S 73 & S 107 & S 197 & $\begin{array}{c}\text { Moyenne } \\
\text { (H) }\end{array}$ & \\
\hline $\begin{array}{l}\text { Phyo } 636 \\
\text { PM } 217 \\
\text { L } 29 \\
\text { CM } 334\end{array}$ & $\begin{array}{l}-0,513 \\
-0,550 \\
-1,703 \\
-1,293\end{array}$ & $\begin{array}{l}-0,593 \\
-0,325 \\
-1,737 \\
-1,195\end{array}$ & $\begin{array}{l}-0,465 \\
-0,178 \\
-1,120 \\
-0,722\end{array}$ & $\begin{array}{l}-0,524 \mathrm{c} \\
-0,351 \mathrm{c} \\
-1,520 \mathrm{a} \\
-1,070 \mathrm{~b}\end{array}$ & $\begin{array}{l}-2,500 \\
-1,025 \\
-3,260 \\
-2,458\end{array}$ & $\begin{array}{l}-2,405 \\
-1,585 \\
-3,820 \\
-2,290\end{array}$ & $\begin{array}{l}-1,290 \\
-0,665 \\
-3,460 \\
-1,875\end{array}$ & $\begin{array}{l}-2,065 \mathrm{~b} \\
-1,092 \mathrm{c} \\
-3,513 \mathrm{a} \\
-2,207 \mathrm{~b}\end{array}$ & $\begin{array}{l}3,94 \\
3,11 \\
2,31 \\
2,06\end{array}$ \\
\hline $\begin{array}{c}\text { Moyenne } \\
\text { des } \\
\text { souches }\end{array}$ & $\begin{array}{c}-1,015 \\
a\end{array}$ & $\begin{array}{c}-0,963 \\
a\end{array}$ & $\begin{array}{c}-0,624 \\
b\end{array}$ & & $\begin{array}{c}-2,311 \\
a b\end{array}$ & $\begin{array}{c}-2,525 \\
\mathrm{a}\end{array}$ & $-1,823$ & & \\
\hline
\end{tabular}

ne permet pas une bonne estimation du critère d'inductibilité : le champignon est souvent complètement bloqué dans sa progression, dès avant le $7^{\mathrm{e}}$ jour à $22^{\circ} \mathrm{C}$, le $5^{\mathrm{e}}$ à $32^{\circ} \mathrm{C}$. Elle ne figure pas dans le tableau 2. Parmi les 3 autres souches, $\mathrm{S} 197$ est la moins inductrice, ce qui confirme les résultats antérieurs (POCHARD \& DAUBEZE, 1980).

On remarque que le processus d'induction n'est pas supprimé à $32^{\circ} \mathrm{C}$ : il est, au contraire accéléré de 2 à 4 fois. « Phyo 636 » est le plus réactif à la température.

\section{Limites d'efficacité de la résistance}

Si l'on poursuit l'analyse au-delà du $7^{\mathrm{e}}$ jour, de très notables différences apparaissent entre les variétés, indépendamment des valeurs de réceptivité et d'inductibilité. L'évolution de la vitesse de nécrose des tiges durant toute la période d'observation est reportée aux figures 1 et 2, à l'exception des valeurs concernant la souche 101 qui n'apportent que très peu d'éléments de discrimination entre les variétés (mise à part la vitesse initiale de nécrose).

On peut remarquer tout d'abord que le passage de $22^{\circ}$ à $32^{\circ} \mathrm{C}$ ne modifie pas fondamentalement le comportement des variétés. On constate seulement l'existence de cinétiques plus rapides. Le classement des souches est inchangé par les hautes températures dans les cas où des différences apparaissent (chez « Phyo 636 » et « L 29 »).

La résistance n'est réellement efficace que si l'induction se poursuit suffisamment longtemps, compte tenu de la vitesse initiale de nécrose et du rythme de décélération, pour aboutir à un blocage de la progression du champignon ou, tout au moins, à des vitesses inférieures à $0,5 \mathrm{~mm}$ par jour. On a reporté au tableau 3 les vitesses les plus faibles observées pendant la totalité de la période de mesure.

Il apparaît que seule la lignée « CM 334 » est complètement résistante dans toutes les situations. «PM 217 » vient en $2^{e}$ position, les minima étant tous inférieurs à $1 \mathrm{~mm}$ par jour, le plus souvent inférieurs à $0,5 \mathrm{~mm}$. Chez « L 29 », la résistance est incomplète pour la souche 197 à $22^{\circ} \mathrm{C}$ et, pour les 3 souches, à $32^{\circ} \mathrm{C}$. La résistance très incomplète de « Phyo 636 » est partout apparente.

Si l'on considère l'effet moyen des souches, on remarque que la vitesse minimale est d'autant plus élevée que la capacité d'induction est plus faible (tabl. 2).
En définitive, c'est la durée de la période de ralentissement quasi-linéaire qui va déterminer l'efficacité. Il est malheureusement très difficile d'établir expérimentalement le moment précis où cet effet cesse. On peut, par contre, mesurer l'écart entre la vitesse initiale et la vitesse minimale (tabl. 3). Ceci pourrait être une mesure de la capacité de résistance des variétés en comparaison. Cependant, la valeur de ce critère change en fonction de la souche et de la température pour une variété donnée.

L'effet particulier des souches ne peut être évité : l'efficience des mécanismes de défense, à quelque niveau que l'on se place, est meilleure vis-à-vis des souches 73 et 107 que de la souche 197. Pour une évaluation globale, on peut considérer la moyenne de ces 3 souches d'origine et de caractéristiques fort différentes.

Il semble possible de trouver un critère qui compense l'effet de la température, minimise les différences entre souches et permette d'attribuer à chaque variété une valeur caractéristique permettant de prévoir les limites de la résistance.

\section{Evaluation du potentiel de résistance}

Nous considérons maintenant, pour chaque variété, la valeur moyenne observée en présence des 3 souches les plus agressives. La $1^{\text {re }}$ partie de la courbe d'évolution de la vitesse de nécrose peut être assimilée à une droite, tout au moins tant que cette vitesse ne descend pas en-dessous de $1 \mathrm{~mm}$ par jour (tabl 4).

Pour simplifier encore, on assimile la totalité du déroulement de la résistance au segment de la droite de régression compris entre l'origine des temps (le jour de l'inoculation) et le temps nécessaire pour atteindre le minimum de vitesse $\left(d_{i}=\right.$ durée de l'induction efficace $) ; Y_{0}$ est l'ordonnée à l'origine (vitesse de nécrose estimée au temps 0 ) et $Y_{\min }$ la vitesse minimum observée au cours des 3 premières semaines.

Une autre origine des temps pourrait être prise, par exemple le $3^{\mathrm{e}}$ jour. Cependant, lors de la comparaison de couples quasi isogéniques sauf pour la résistance à Phytophthora, on constate, en prolongeant les courbes relatives aux 
Vitesse de nécrose de la tige

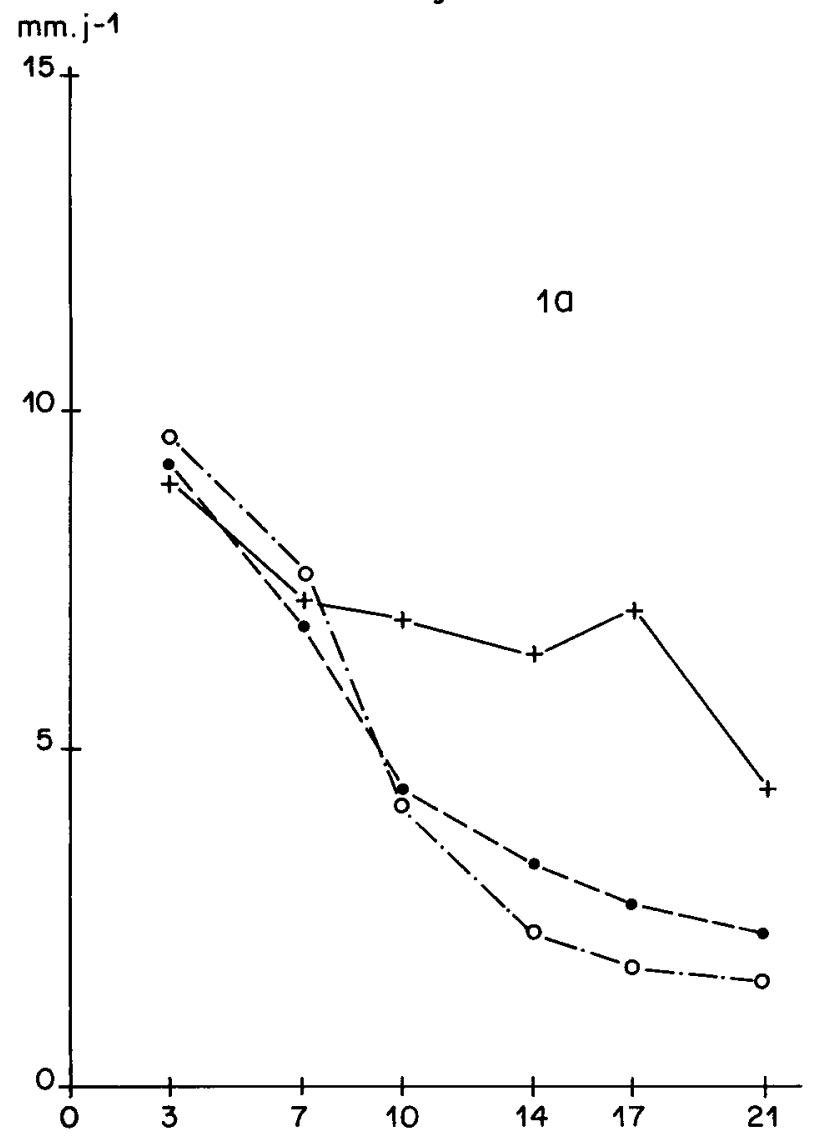

$\mathrm{mm} . \mathrm{j}-1$

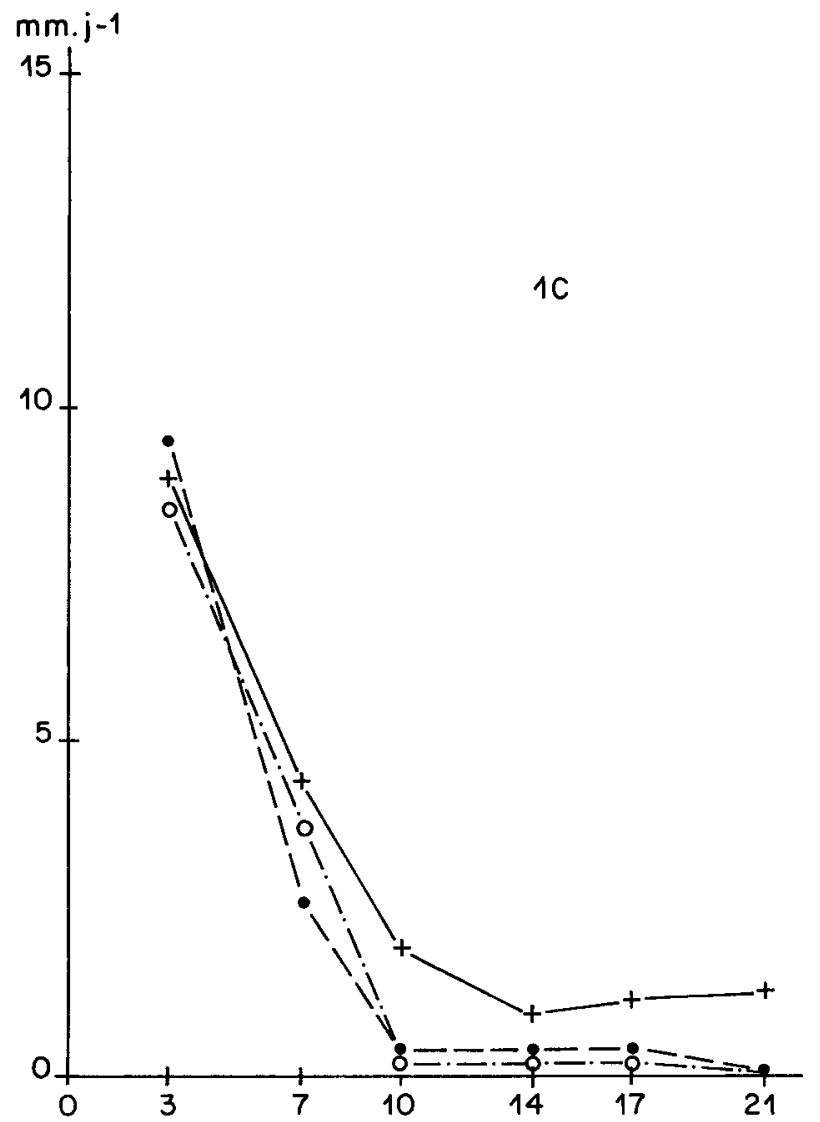

Figure 1

Evolution de la vitesse de nécrose de la tige en fonction du temps: essai à $22^{\circ} \mathrm{C}$.

$1 a=$ Phyo 636; $1 b=P M 217 ; 1 c=L 29 ; 1 d=C M 334$

O-•-O Souche 73 ; •-• Souche 107 ; + - + Souche 197.

Revue d'Agronomie $n^{\circ}$ 4-1983

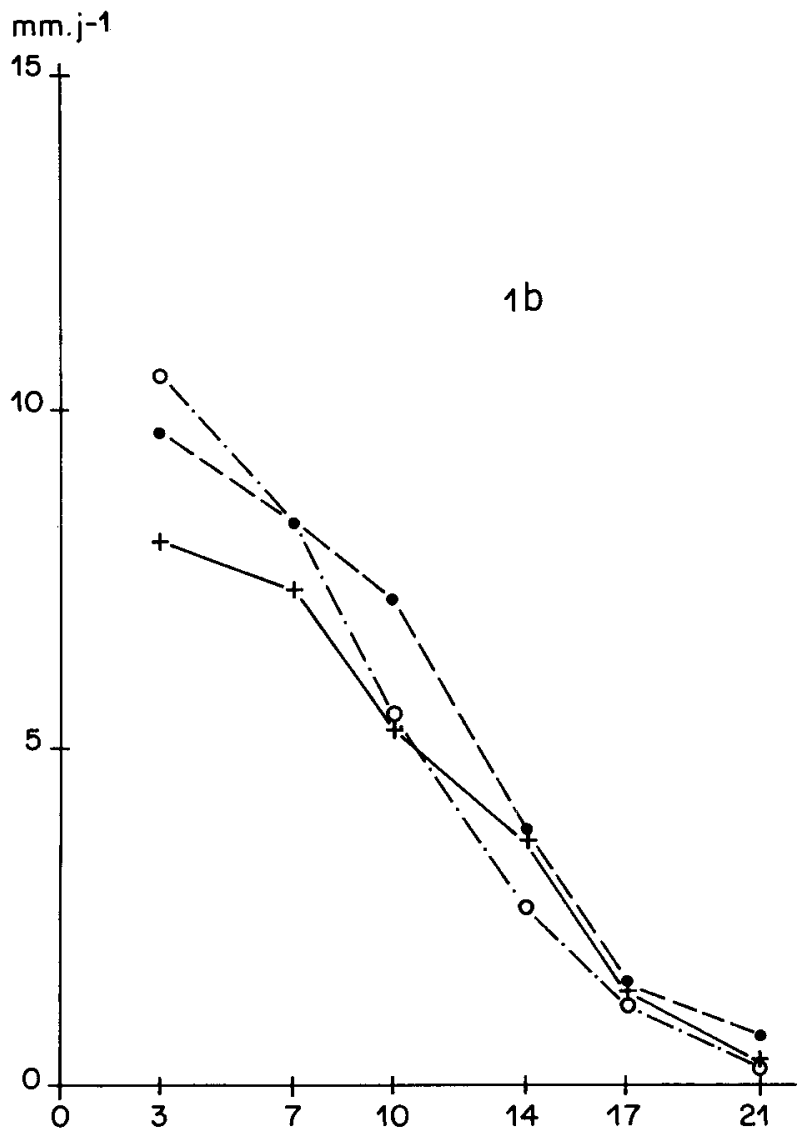

$\mathrm{mm} . \mathrm{j}-1$

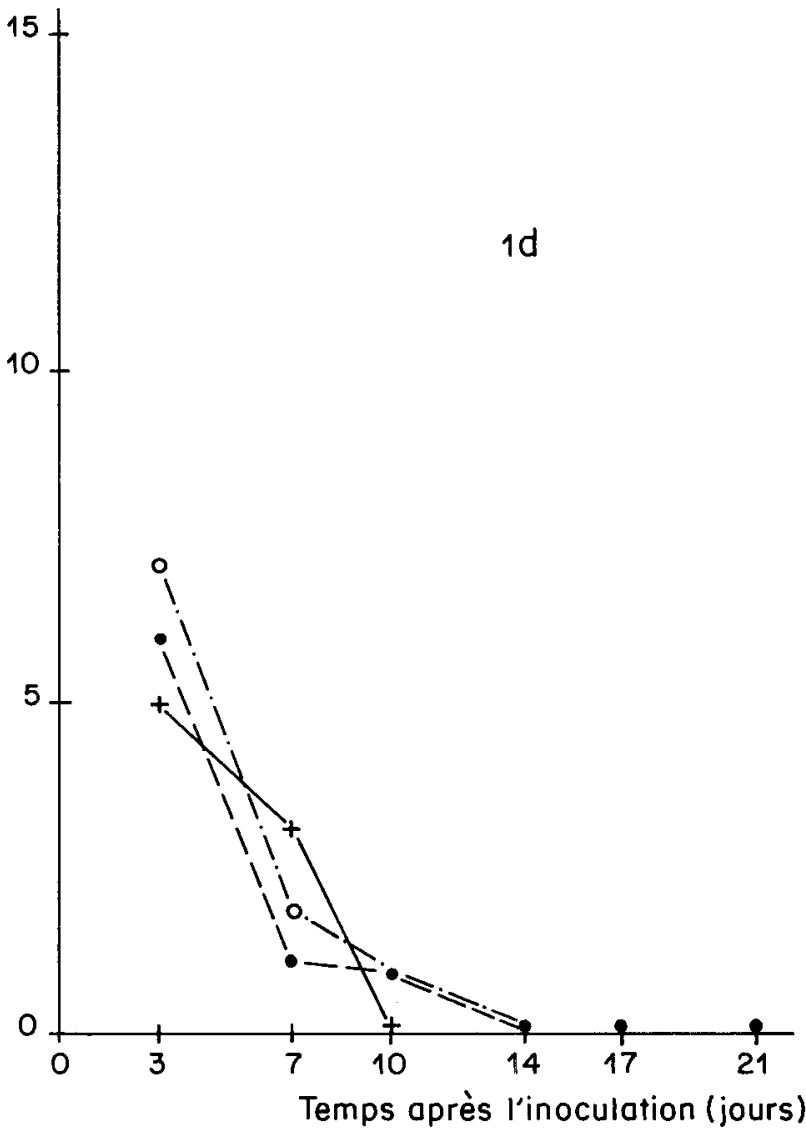

Evolution of the speed of stem necrosis during the 3 weeks following inoculation, at $22^{\circ} \mathrm{C}$.

Varieties : $1 a=$ Phyo $636 ; 1 b=P M 217 ; 1 c=L 29 ; 1 d=C M 334$ Strains : 73 ०-॰-0; $107 \bullet--\bullet ; 197+-+$ 
Vitesse de nécrose de la tige
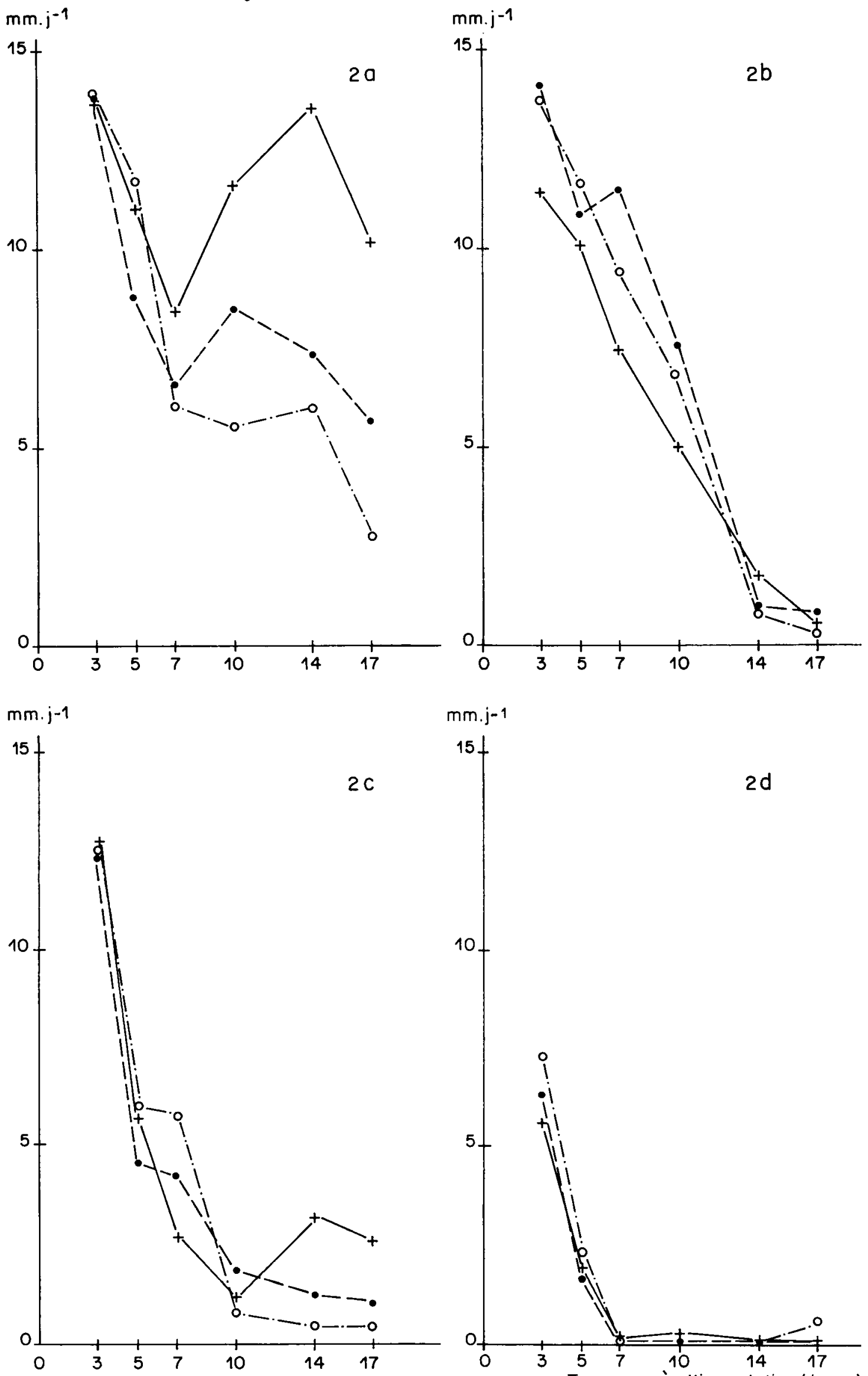

$m m . j-1$

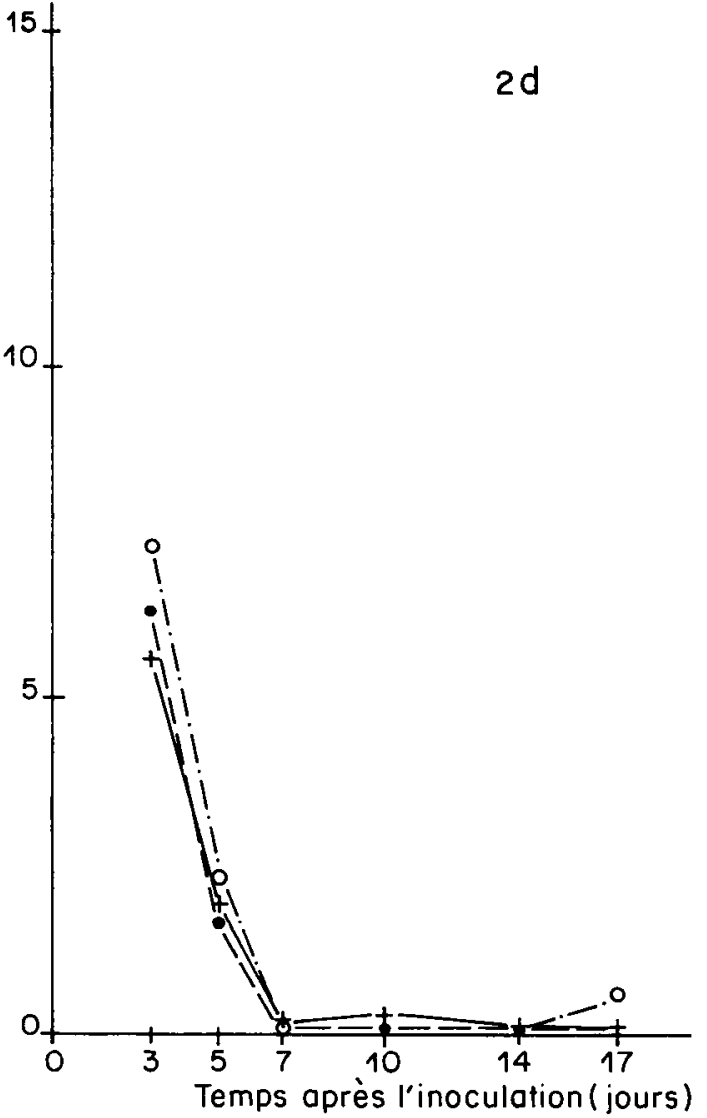

Figure 2

Evolution de la vitesse de nécrose de la tige en fonction du temps: essai dे $32^{\circ} \mathrm{C}$.

Evolution of the speed of stem necrosis at $32^{\circ} \mathrm{C}$. Mêmes symboles que la figure 1. 
TABLEAU 3

Limites d'efficacité de la résistance

$A$ : minimum de vitesse de nécrose observé pendant la période de mesures (mm.jour ${ }^{-1}$ )

$B$ : écart entre la vitesse initiale et le minimum (CM 334 exclu)

Limits of the efficiency of resistance mechanisms

A : minimum speed of stem necrosis during the period of observation (mm.day ${ }^{-l}$ )

$B$ : difference between the initial and the minimum speed of necrosis (CM 334 excluded)

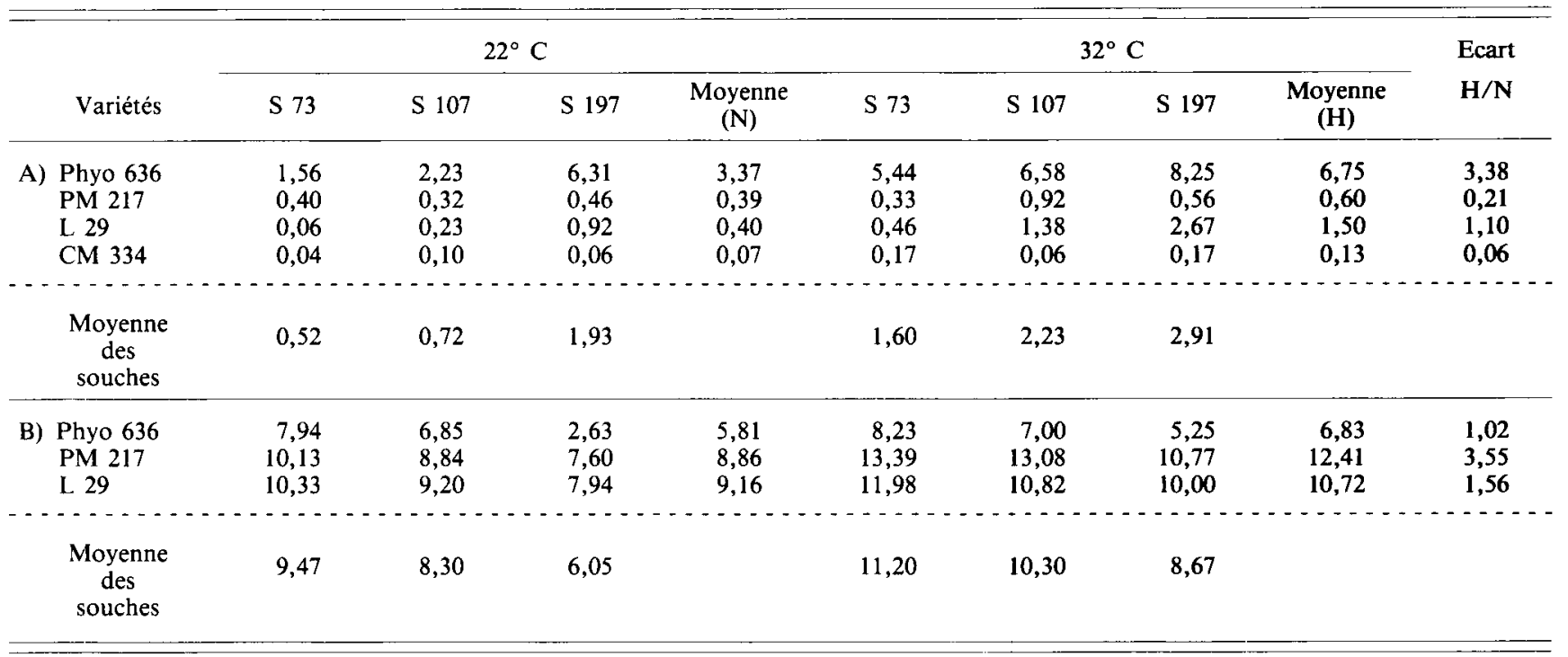

TABLEAU 4

Représentation linéaire du déroulement de la résistance (moyenne des 3 souches les plus agressives) Le potentiel de résistance $P_{R}$ est estimé par le produit $\left(Y_{0}-Y_{\text {min }}\right) d_{i}$ (voir texte)

( ) nombre de périodes prises en compte dans la régression de y (vitesse de nécrose) sur $x$ (le temps)

Linear representation of the development of resistance (mean of the 3 most aggressive strains). Resistance potential or $P_{R}$ is estimated by the product $\left(y_{0}-y_{\text {min }}\right) d_{i}$ (see text) 1 ) number of periods used in the regression $y$ (speed of necrosis) on $x$ (time)

\begin{tabular}{|c|c|c|c|c|c|c|}
\hline \multirow{2}{*}{$\begin{array}{l}\text { Variétés } \\
\text { Régression }\end{array}$} & \multicolumn{2}{|c|}{ Phyo 636} & \multicolumn{2}{|c|}{ PM 217} & \multicolumn{2}{|c|}{ L 29} \\
\hline & $22^{\circ}$ & $32^{\circ}$ & $22^{\circ}$ & $32^{\circ}$ & $22^{\circ}$ & $32^{\circ}$ \\
\hline b y/x & $-0,484(4)$ & $-1,660(3)$ & $-0,585(5)$ & $-1,056(5)$ & $-1,273(3)$ & $-2,040(4)$ \\
\hline$y_{0}$ & 10,4 & 18,3 & 11,6 & 16,4 & 13,1 & 17,6 \\
\hline$y \min$. & 3,8 & 6,8 & 0,4 & 0,6 & 0,4 & 1,5 \\
\hline $\mathrm{Ve}$ & 0,1950 & 0,4374 & 0,1790 & 0,2816 & 0,7143 & 4,322 \\
\hline F régr. & 78,0 & 50,4 & 234,9 & 296,0 & 56,0 & 11,6 \\
\hline Prob. & $<0,05$ & $<0,10$ & $<0,001$ & $<0,001$ & $<0,10$ & NS \\
\hline & 6,6 & 11,5 & 11,2 & 15,8 & 12,7 & 16,1 \\
\hline $\begin{array}{l}\mathrm{y}_{\mathrm{o}} \mathrm{y}_{\min } \\
\mathrm{d}_{\mathrm{j}} \text { (jours) }\end{array}$ & 13,5 & 7,0 & 19,1 & 14,9 & 9,9 & 7,9 \\
\hline $\mathrm{P}_{\mathrm{R}}$ & \multirow{2}{*}{\multicolumn{2}{|c|}{85}} & \multirow{2}{*}{\multicolumn{2}{|c|}{236}} & 126 & 127 \\
\hline$P_{R}^{K}$ moyen & & & & & \multicolumn{2}{|c|}{126} \\
\hline
\end{tabular}

partenaires sensibles et résistants, que celles-ci semblent partir d'un même point situé non loin du moment de l'inoculation (PoCHARD et al., 1976, 1981).

On remarque que le produit $\left(\mathrm{Y}_{0}-\mathrm{Y}_{\mathrm{min}}\right) \mathrm{d}_{\mathrm{i}}$ ne varie que très peu avec la température et pourrait représenter l'un des éléments de la résistance responsable de la durée limitée d'une induction efficace. Nous avons appelé cet élément " potentiel de résistance» ou $\mathrm{P}_{\mathrm{R}}$.

La représentation graphique des phénomènes est donnée sur la figure 3.

Il apparaît que « Phyo 636 » a conservé moins de la moitié du potentiel de son géniteur d'origine «PM 217 ». « L 29 " ne possède pas non plus des caractéristiques très remarquables dans ce domaine.

Pour tester $\mathrm{P}_{\mathrm{R}}$ en tant qu'indice caractéristique de chaque variété, on a calculé, à partir des données figurant aux tableaux $1 \& 2$, le minimum de vitesse, $Y_{\min }$, donné par la relation $\mathrm{Y}_{\min }=\mathrm{Y}_{0}-\sqrt{-\mathrm{b} . \mathrm{P}_{\mathrm{R}}}$ (b étant le coefficient de régression de y sur le temps pour chaque couple variété-souche) (tabl. 5).

Malgré l'imprécision de l'estimation de b, on constate que les valeurs ainsi calculées ne s'éloignent pas considérablement des valeurs observées (tabl. 3). Les prévisions sont en général un peu au-dessus de la réalité spécialement pour « PM 217 » à $22^{\circ} \mathrm{C}$. Chez cette variété, l'induction de résistance apparaît souvent un peu retardée (cf. tabl. $2 \& 4$ ).

\section{E. Accumulation du capsidiol}

Il a été montré que l'accumulation de capsidiol dans les tiges de piment contaminées par décapitation était la plus intense au $4^{\mathrm{e}}$ jour sur quelques mm en avant du front des 


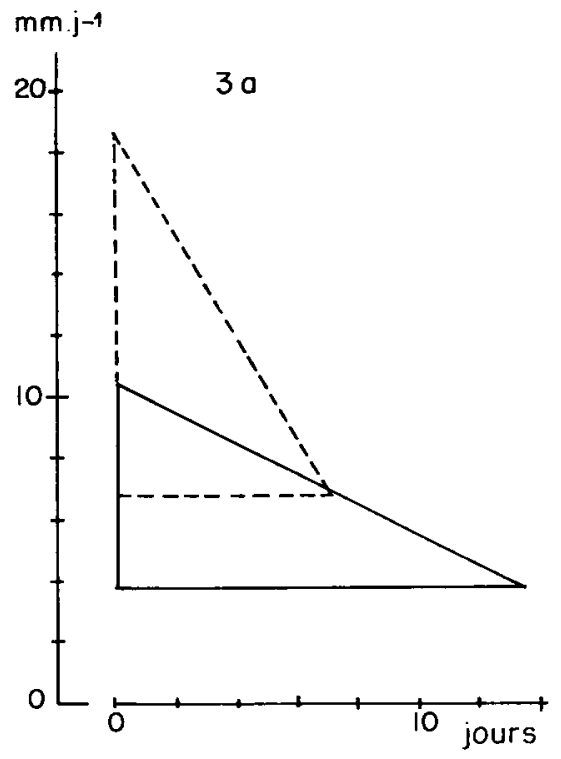

$3 b$

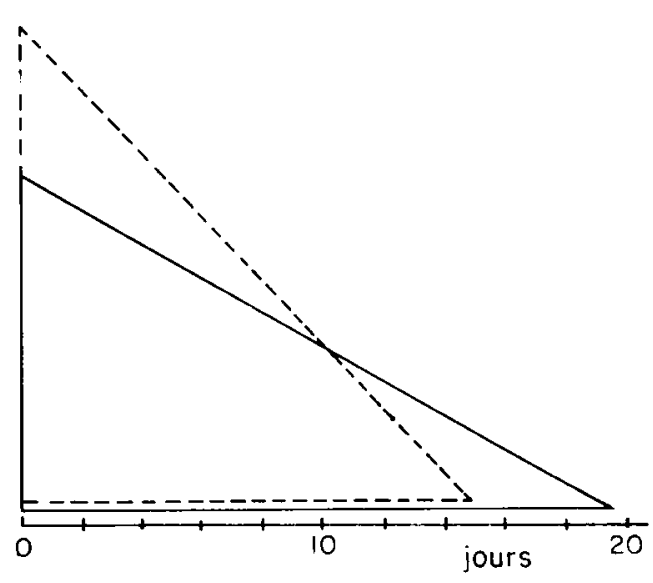

$3 c$

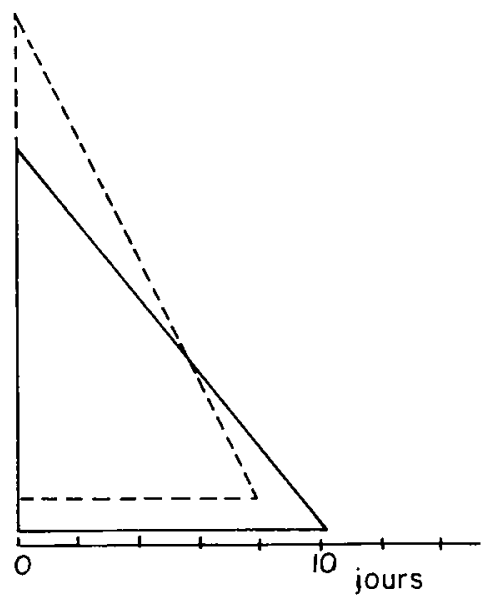

Figure 3

Représentation schématique de la relation entre la plante et l'agent pathogène : évaluation du potentiel de résistance $P_{R}$. $L$ 'évolution de la vitesse en fonction du temps est assimilée à un segment de droite construit de $y=y_{o} \grave{a} y=y_{\text {min }} \grave{a}$ partir de la régression linéaire correspondant aux 3 à 5 premières mesures (voir tabl. 4).

Moyenne des 3 souches les plus agressives $; P_{R}=2$ fois la surface du triangle ; _ : $22^{\circ} \mathrm{C} ;---32^{\circ} \mathrm{C} ; a, b, c$, comme la figure 1 .

Schematic representation of the relation between plant and pathogen : evaluation of resistance potential $P_{R}$.

The evolution of the speed is assimilated to a linear segment from $y=y_{o}$ to $y=y_{\min }$ extension of the linear regression corresponding to the 3 to 5 first measures (see tabl. 4).

Mean of the 3 most aggressive strains $; P_{R}=2$ times the area of the triangle; _ $: 22^{\circ} \mathrm{C} ;--: 32^{\circ} \mathrm{C} ; a, b, c$, as in figure 1.

TABLEAU 5

Limites de la résistance calculées pour un potentiel $P_{R}$ constant.

Evaluation de la vitesse minimum de nécrose. Les paramètres utilisés sont : $y_{3}$ (vitesse estimée au $3^{e}$ jour : tabl. 1) et $b_{3-7}$ décélération de la vitesse entre le $3^{e}$ et le $7^{e}$ jour: tabl. 2)

Limits of the resistance computed for a constant potential $\left(P_{R}\right)$.

Evaluation of the minimum speed of necrosis. The parameters used in the computations are $y_{3}$ (estimated speed on the 3 rd day, tabl. 1 ) and $b_{3-7}$ (deceleration of the speed between the $3 r d$ and the 7 th day, tabl. 2)

\begin{tabular}{|c|c|c|c|c|c|c|c|c|c|}
\hline \multirow[b]{2}{*}{ Variétés } & \multirow[b]{2}{*}{$\mathbf{P}_{\mathrm{R}}$} & \multicolumn{2}{|c|}{$22^{\circ} \mathrm{C}$} & \multicolumn{6}{|c|}{$32^{\circ} \mathrm{C}$} \\
\hline & & S 73 & S 107 & S 197 & Moyenne & S 73 & S 107 & S 197 & Moyenne \\
\hline $\begin{array}{l}\text { Phyo } 636 \\
\text { PM } 217 \\
\text { L } 29\end{array}$ & $\begin{array}{r}85 \\
225 \\
126\end{array}$ & $\begin{array}{l}4,44 \\
1,06 \\
0,85\end{array}$ & $\begin{array}{r}3,76 \\
2,14 \\
-\quad 0,05\end{array}$ & $\begin{array}{l}4,05 \\
3,92 \\
0,14\end{array}$ & $\begin{array}{l}4,08 \\
2,37 \\
0,31\end{array}$ & $\begin{array}{l}6,59 \\
1,61 \\
1,45\end{array}$ & $\begin{array}{r}6,48 \\
-\quad 0,13 \\
1,74\end{array}$ & $\begin{array}{l}6,90 \\
1,09 \\
2,17\end{array}$ & $\begin{array}{l}6,65 \\
0,86 \\
1,95\end{array}$ \\
\hline
\end{tabular}

hyphes mycéliennes (MOLOT et al., 1981). Il faut donc estimer la position des hyphes par rapport au front de nécrose et prélever un disque de tige dans la zone non encore envahie pour estimer la quantité de capsidiol par un test biologique. La comparaison de « L 29 » et « CM 334 » aux 2 variétés déjà bien connues fait apparaître 2 particularités. Tout d'abord, chez «CM 334 », le front d'avancée des hyphes ne précède que de $0,5 \mathrm{~mm}$ environ le front de nécrose. Chez les autres variétés, la marge est de $4 \mathrm{~mm}$ environ. Chez le témoin sensible «Yolo Wonder », elle est proche de $10 \mathrm{~mm}$ (tabl. 6).

L'autre particularité concerne l'accumulation du capsidiol : elle est notable chez «PM 217 » et «CM 334 »; par contre, chez « L 29 », elle est du même ordre de grandeur que celle du témoin sensible. On peut donc penser que les différents géniteurs de résistance ne mettent pas en jeu les mêmes mécanismes biochimiques.

\section{DISCUSSION - CONCLUSION}

Sachant que, dans les conditions du Mexique, le matériel de type «PM 217 » et «Phyo 636 » manifeste une résistance insuffisante alors que les lignées « L 29 » et surtout "Criollo de Morelos 334 » présentent une résistance effective (GUERRERO-MORENO \& LABORDE, 1980 et comm. pers.), nous nous sommes demandé en quoi ces variétés différaient de celles dont nous avons cherché, de longue date, à exploiter la résistance. Les questions posées étaient de 3 ordres : ces variétés diffèrent-elles des nôtres par un ou plusieurs des éléments de résistance précédemment décrits (Pochard \& Daubeze, 1980) ? Ont-elles un spectre d'activité différent vis-à-vis des souches du champignon ? Sontelles plus stables vis-à-vis des températures élevées ? Des éléments de réponse à ces 3 questions sont maintenant réunis. 
TABLEAU 6

Accumulation de capsidiol dans la tige de piment 4 jours après l'inoculation

Capsidiol accumulation in the pepper stem 4 days after inoculation

\begin{tabular}{|c|c|c|c|c|c|}
\hline & Yolo W & Phyo 636 & PM 217 & L 29 & CM 334 \\
\hline $\begin{array}{l}\mathbf{L} \\
1 \\
\mathrm{C}\end{array}$ & $\begin{array}{l}42 a \\
10,6 a \\
14 a\end{array}$ & $\begin{array}{cc}27 & b \\
3,4 & b \\
18 & b\end{array}$ & $\begin{array}{c}24 \mathrm{bc} \\
4,0 \mathrm{~b} \\
22 \mathrm{c}\end{array}$ & $\begin{array}{cc}21 & \mathrm{c} \\
4,0 \mathrm{~b} \\
13 & \mathrm{a}\end{array}$ & $\begin{array}{l}16 \mathrm{~d} \\
0,6 \mathrm{c} \\
21 \quad \mathrm{bc}\end{array}$ \\
\hline
\end{tabular}

$c=$ quantité de capsidiol devant le front de progression des hyphes mycéliennes, évaluée par le diamètre de la zone d'inhibition de Cladosporium herbarum. $L=$ longueur de la nécrose externe de la tige en $\mathrm{mm}$.

$l=$ avance des hyphes par rapport au front de nécrose en $\mathrm{mm}$.

Comparaison par le test de Duncan au seuil 0,01.

$c=$ quantity of capsidiol in advance of the hyphae evaluated by the diameter of inhibition spot of Cladosporium herbarum (mm).

$L=$ length of external sterm necrosis. $l=$ advance of hyphae with regard to the necrosis, in $\mathrm{mm}$.

Comparison by the Duncan test, at the level 0,01.

La lignée « Criollo de Morelos 334 » est remarquable par la très faible vitesse initiale de progression du champignon dans la tige. D'après la terminologie que nous avons proposée, cette variété a une faible réceptivité. Cette réceptivité réduite est efficace vis-à-vis de toutes les souches essayées (choisies aussi différentes que possible) d'une manière d'autant plus nette que la souche est plus agressive (souche 107) ou moins bonne inductrice de résistance (S 197). Ceci est valable pour d'autres souches de la zone méditerranéenne. Des essais récents conduits à l'aide de 12 souches extrêmement agressives isolées en Turquie (données non publiées) montrent l'aspect général, non spécifique, de ce comportement. Il n'est donc pas nécessaire de supposer qu'il existe au Mexique des souches d'une spécificité de virulence différente de celles que l'on rencontre en Europe.

Chez « CM 334 », la réceptivité n'est pas modifiée par une augmentation de la température de $22^{\circ}$ à $32^{\circ} \mathrm{C}$. Chez tous les autres matériels essayés à ce jour, sensibles ou partiellement résistants à Phytophthora, la vitesse est augmentée de 30 à 90 p. 100 en fonction du matériel et de la souche employés. Cette insensibilité à la température, dans la gamme choisie, a pu être vérifiée dans d'autres essais. Elle se transmet bien par la voie héréditaire, quel que soit le sens du croisement (données non publiées). Un comportement aussi original mériterait d'être approfondi. Il permet de mieux comprendre les performances exceptionnelles de « Criollo de Morelos 334 » dans des zones de culture où la température doit souvent dépasser $30^{\circ} \mathrm{C}$ au niveau de la plante.

La lignée « L 29 » qui n'a aucune caractéristique remarquable au niveau de la réceptivité, présente, par contre, une excellente aptitude à l'induction : elle possède, d'après notre terminologie, une très bonne inductibilité. La décélération de la vitesse de progression du champignon est plus intense chez cette variété que chez toute autre. Ce comportement se manifeste avec les 4 souches essayées. Il apparaît tout aussi nettement à $32^{\circ} \mathrm{C}$ qu'à $22^{\circ} \mathrm{C}$, la décélération étant, chez toutes les variétés, plus rapide à haute température. Il est intéressant de remarquer que « L 29 » montre aussi la réponse la plus intense, au niveau du feuillage, aux éliciteurs extraits du champignon : la résistance induite est plus importante que celle que l'on peut déceler chez «Phyo 636 », « PM 217 » et « CM 334 ». Cependant, dans les feuilles comme dans les tiges, ce comportement n'est pas associé à une accumulation de capsidiol (MoLot et al., 1983).

Le géniteur « PM 217 » garde cependant un grand inté- rêt. On a pu montrer en effet qu'il possédait une caractéristique très favorable : l'aptitude à maintenir une induction efficace de résistance pendant une longue période, de l'ordre de 3 semaines, alors que cette induction semble plus fugace chez les autres variétés, tout particulièrement « Phyo 636 ». La notion de stabilité de la résistance apparaît donc à nouveau ( $3^{\mathrm{e}}$ composante de la résistance définie en 1980 ). L'utilisation de souches très agressives à 2 températures différentes révèle un phénomène qui était passé inaperçu. Même chez la variété la moins résistante, « Phyo 636 », il existe toujours une phase de ralentissement linéaire de la vitesse de nécrose de la tige. Ce ralentissement cesse après un temps déterminé comme si la plante avait épuisé un « capital de résistance » ou se trouvait dans l'incapacité de mettre en œuvre un processus efficace pour contenir la progression du champignon.

Nous définissons un nouveau critère : le « potentiel de résistance ». Dans les tests sur tige décapitée, il est évalué par le produit de 2 termes dont l'un est la durée estimée de la phase de ralentissement linéaire de la progression du champignon. La valeur du potentiel de résistance des tiges adultes (les 3 souches les plus agressives étant confondues) n'est pratiquement pas modifiée par la température. Connaissant cette valeur, il devient possible de prévoir si la résistance, en présence d'une souche nouvelle, sera totalement efficace ou, au contraire, incomplète et instable. Il suffit de connaître la vitesse initiale de pénétration (mesure de la réceptivité) et le coefficient de décélération (mesure de l'inductibilité).

La notion de potentiel de résistance ne semble pas limitée aux tiges de plantes adultes. En inoculant les racines de très jeunes plantes par une suspension de zoospores, on montre que « PM 217 » est nettement plus résistant à l'envahissement et à la destruction par le champignon que « $L$ 29 ", lui-même plus résistant que « Phyo 636 » (PocHARD \& DAUBEZE, à paraître). « CM 334 » est encore le meilleur, mais pas très supérieur à « PM 217 », malgré sa position très favorable en ce qui concerne la « réceptivité ».

On peut espérer construire une résistance peu spécifique et d'un niveau extrêmement élevé en recombinant les genes utiles portés par les 3 principales sources de résistance décrites dans ce travail.

Reçu le 8 juillet 1982. Accepté le 30 novembre 1982.

\section{REMERCIEMENTS}

Nous tenons à exprimer nos remerciements au Professeur J. CHEVAUGEON qui a bien voulu lire le manuscrit et nous aider de ses précieux conseils. 


\section{RÉFÉRENCES BIBLIOGRAPHIQUES}

Clerjean M., Pitrat M., Nourrisseau J.-G., 1976. La résistance du piment (Capsicum annuum) à Phytophthora capsici. IV. Etude de l'agressivité de divers isolats au niveau des feuilles, des tiges et du collet des plantes sensibles et résistantes. Ann. Phytopathol, 8 (4), 411-423.

Guerrero-Moreno A., Laborde J.-A., 1980. Current status of pepper breeding for resistance to Phytophthora capsici in Mexico. Eucarpia Capsicum Working Group, 4th Meeting, 14-15 October, 1980, Wageningen, p. 52-56.

Kimble K.A., Grogan R.G., 1960. Resistance to Phytophthora root rot in pepper. Plant Dis. Rep., 44, (11), 872-873.

Molot P.M., Clerjean M., Nourrisseau J.G., Ricci P., 1976. La résistance du piment (Capsicum annuum) à Phytophthora capsici. III. Etude sur extraits de tiges sensibles et résistantes, du pouvoir antifongique induit par la contamination. Ann. Phytopathol., 8, (4), 399-409.

Molot P.M., Clerjeau M., Mas P., Ricci P., 1977. Rôle du capsidiol dans la résistance génétique du piment (Capsicum annuum) au Phytophthora capsici. "Capsicum 77 ", C.R. $3^{e}$ Congres Eucarpia, 5-7 juil., Edit. E. Pochard, I.N.R.A. 84140 Montfavet-Avignon, France, 137-146.

Molot P.M., Mas P., Conus M., Ferrière H., Ricci P., 1981. Relations between capsidiol concentration, speed of fungal invasion and level of induced resistance in cultivars of pepper (Capsicum annuum) susceptible or resistant to Phytophthora capsici. Physiol. Plant Pathol., 18, 379-389.
Molot M., Pochard E., Mas P., 1983. La résistance du piment (Capsicum annuum L.) à Phytophthora capsici Leon. XI. Réponse de 5 lignées de piment à une " fraction élicitrice "; influence de la dose d'éliciteur et efficacité de la protection induite vis-à-vis de plusieurs souches du parasite. Agronomie, 3 (4), 327-332.

Pochard E., Clerjeau M., Pitrat M., 1976. La résistance du piment (Capsicum annuum) à Phytophthora capsici. I. Mise en évidence d'une induction progressive de la résistance. Ann. Amélior, Plant., 26 (1), 35-50.

Pochard E., Daubèze A.-M., 1980. Recherche et évaluation des composantes d'une résistance polygénique : la résistance du piment à Phytophthora capsici. Ann. Amélior. Plant., 30 (4), 377-398.

Pochard E., Chalal N., Marchoux G., 1981. Effect spécifique de 3 virus sur l'expression de la résistance à une maladie cryptogamique du piment due à Phytophthora capsici Leon. Agronomie, 1 (7), 521-526.

Smith P.G., Kimble K.A., Grogan R.G., Millet A.H., 1967. Inheritance of resistance in peppers to Phytophthora root rot. Phytopatho$\log y, 57,377-379$. 